\title{
SÉMIOTIQUE DU LIMINAIRE CAMUSIEN
}

\author{
Richard-Laurent Barnett*
}

Un livre ne commence ni ne finit: tout au plus fait-il semblant.

Mallarmé

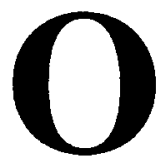

utre qu'il nous dispense d'inaugurer, l'exergue mallarméen - lequel fait fonction de préface à la fois pré-textuelle et para-textuelle définit bien la feinte qui réside au fondement de tout début romanesque: à savoir qu'il n'y a lieu de faire semblant que dans la mesure où une absence, un blanc - ce vide qui le précède - au texte se noue. Par son incipit, ${ }^{1}$ le texte feint de commencer, en dissimulant ce qui en lui n'est pas lui, ne lui vient que d'ailleurs: car ce n'est que de l'effacement des discours du réel qu'il peut, fondant sa cohérence, accéder lui-même à ce réel illusoire, surajouté, qu'est toujours la littérature, lors même qu'elle fait vrai.

Rompant avec le non-dit, en ce seuil qui l'en sépare, l'incipit s'arrache à la "prose du monde", et ne se donne pour vrai qu'en offusquant sa différence. Feinte où transparaît la difficulté de tout commencement, la tension qui résulte de cette exigence contradictoire: fonctionner comme un réel, au moment même où l'on s'en éloigne pour s'engager dans la fiction.

* Marygrove College (Detroit, Michigan). 
On le sait, il est en écriture qui s'acharne à se faire passer pour vraie, à se dédire comme fiction; cette écriture, que l'on dit réaliste, si elle appartient à une époque bien définie, ne se confond pas moins dans son principe avec le genre romanesque tout entier. Elle relève de ce que, tour à tour, Machery, Barthes, Ricardou et Riffaterre ont dénoncé sous le terme d'illusion référentielle: la croyance en une référence extérieure au texte qui serait à l'origine d'une activité narrative soumise à l'ordre de la représentation, là où il ne faut voir que production d'un effet de réalité. Le narrateur, loin de partir d'une différence attestable en dehors du volume textuel, la produit littéralement par la médiation de son récit.

Cette illusion est au principe de la production de l'intérêt romanesque: le roman n'est en mesure d'intéresser qu'à la condition d'effacer, autant que possible, son opération matérielle, littérale. Il ne fonctionne au mieux qu'en masquant ce qui en lui relève de l'artifice, et peut porter préjudice à la transparence de l'objet narré.

Nous pouvons dès lors, à la suite de Grivel, ${ }^{2}$ formuler un ensemble de propositions, situant notre analyse, mais qui, dans leur généralité, dépassent les limites imposées par ce type de démarche:

- le roman, considéré sous l'angle de sa communication, n'existe pas en dehors de l'intérêt qu'il suscite;

- le roman ne produit cet intérêt que par la fabulation: il est une histoire;

- cette fabulation est toujours fabuleuse, extraordinaire: ce n'est qu'en s'écartant de l'ordinaire que le récit parvient à s'instaurer; ce n'est qu'en renouvelant l'imprévu que le récit parvient à se développer;

- c'est avant tout en tant que négativité, plus précisément sous forme de malheur, que se présente cet extraordinaire. Le bonheur, c'est l'inénarrable ( $c f$. Stendhal).

Attendu que "le roman ne comprend que l'élaboration de son commencement", ${ }^{3}$ et qu'il n'existe que par lui, que sur la base de son effet produit, il est impératif de cerner la façon dont le texte se réalise au point de son entrée, les moyens qu'il met en oeuvre pour son actualisation qui, aussi bien, est sa mise en réalité. 


\section{II}

Aujourd'hui, maman est morte. Ou peut-être hier, je ne sais pas. J'ai reçu un télégramme de l'asile: "Mère décédée. Enterrement demain. Sentiments distingués." Cela ne veut rien dire. C'était peut-être hier. $^{4}$

Il y a tout lieu de croire, à première vue, que l'agencement manifesté par cet énoncé romanesque relève de l'illusion réaliste. Toutefois, il pourrait bien n'y participer que de façon problématique, marquant plutôt l'impossibilité de rompre avec le processus de "vraisemblabilisation". Auquel cas, on aurait tort de ne voir en lui que soumission au code dominant du texte. La présente analyse se donne pour but de repérer l'effet spécifique que produit cet incipit. Un tel repérage ne peut s'effectuer sans établir l'intertexte formel de l'incipit romanesque - travail dont Grivel s'est fait l'opérateur.

Que le roman soit uniquement et par-dessus tout production d'intérêt, qu'il ne suscite cet intérêt qu'en provoquant de l'inattendu et de l'extraordinaire sous leur forme négative (malheur), signifie "qu'il n'affiche pas globalement, immédiatement son sens", du fait même qu'il le produit. Aussi l'initiation du roman comprend-elle traditionnellement "l'exposition de la situation de base", 6 un avant-récit où sont distribués les termes du texte, sur fond duquel "l' amorce" permet l'intrusion de l'extraordinaire, dont l'intérêt se mesure à son degré d'imprévisibilité. Mais, on le sait, le roman ne débute pas nécessairement par son exposition, et nombreux sont les romans qui, déjouant la prévisibilité de l'exposition, commencent ex abrupto, et renforcent ainsi leur effet d'imprévu.

Tel est, en apparence, le cas de l'incipit qui nous occupe, ne s'ouvrant pas sur un avant-récit, mais affirmant violemment l'immédiateté de son (ses) sens: "Aujourd' hui, maman est morte".

Dès la première phrase le lecteur se heurte à l'extraordinaire: ${ }^{9}$ l'information s'actualise sans détour ni délai, le malheur de la mort s'installe d'emblée, sans se fonder sur des données contextuelles. Il ne perturbe pas une ordonnance première, ne brise qu'un silence.

Aussi la nomination des personnages (lieux et temps), traditionnellement disséminée à travers l'exposition, se réalise-t-elle dans l'amorce même de l'extraordinaire. L'événement surgit avant que son actant principal ne prenne consistance, et voue celui-ci à ne figurer dans le récit que rétrospectivement. La mort elle-même, l'événement par lequel s'accomplit l'extraordinaire, ne paraît vraisemblable qu'à condition d'être située dans le temps. Dire “aujourd'hui", 
c'est pourvoir l'extraordinaire d'une temporalité propre et spécifique, en fonction d'une mimésis du temps réel.

Outre leur soi-disant fonction comme indices de coordination, les unités "aujourd'hui" et "maman" désignent une relation romanesque: non seulement elles composent l'extraordinaire, mais elles révèlent encore celui par qui le récit est réalisé, rendu (au) réel. "Il importe que l'histoire passe pour être dite: c'est de cette dictée qu'elle trouve sa garantie" ${ }^{\text {"10 }}$. Le narrateur anonyme maintient le récit sous les apparences du discours, il n'est là que pour accréditer discrètement la vérité de l'énoncé: 'témoin, confident, observateur, sa présence change le texte en inattaquable mimésis" ", par l'instauration d'une focalisation fixe. Le narrateur (implicite) fonde la vraisemblance de son énoncé sur le code mimétique de l'écriture (auto-) biographique, s'effectuant "à la première personne du parfait, forme autobiographique par excellence" ${ }^{\prime 2}$.

Alors même que l'incipit semble répondre aux exigences réalistes de la vraisemblance, un glissement s'opère qui, tout en instaurant le sens définitif de la première phrase, dément le bien-fondé de l'indication temporelle, et démunit, par voie de conséquence, l'instance qui la supporte: "Ou peut-être hier, je ne sais pas."

Cette phrase lâchée, c'est tout le potentiel herméneutique de l'extraordinaire qui est gommé, vu que celui-ci mettait en place un événement susceptible de développements analeptiques (circonstances de la mort, vie de la mère, ainsi de suite), et que le récit n'exploite pas, laisse pour compte. Ecartant l'extraordinaire, il s'installe dans le creux libéré par cette mise à l'écart momentanée. L'événement est dit sans être doublé de sa signification émotionnelle. Non seulement ce glissement affecte l'énoncé premier, en lui enlevant son aptitude à "permettre le récit", 13 mais il porte atteinte à l'énonciation elle-même: par l'aveu de son ignorance, le narrateur entame la distribution légitimée du vrai, en laquelle se fonde l'énoncé réaliste. C'est qu'au texte, il est quelque chose de plus nécessaire que sa vraisemblance: l'irréversibilité de sa formulation, contrainte qui fait qu'on n'y peut changer le moindre mot: or celui-ci est fait précisément de continuelles retouches.

A peine ébauché, le récit se dévoile par le démenti de sa stabilité initiale, provoquant le flottement du syntagme événementiel ("maman est morte"), son décrochage temporel.

En ce re-commencement, il y a lieu de discerner une double énigme: la première porte sur la localisation temporelle de l'événement, la seconde sur les causes de cette première énigme. L'étape prochaine du récit ne vise qu'à l'établissement des raisons qui ont mené à l'hésitation, au tiraillement aujourd'hui/hier, et ne cherche nullement à résoudre celle-ci. 
"Contraint de cesser d'intriguer pour exposer", 14 le narrateur, dans un souci de communication, redit ce qu'il sait: "J'ai reçu un télégramme de l'asile". Cette régression temporelle ne présente aucune marque de justification logique: juxtaposée à la précédente, elle n'actualise aucune cheville d'exposition (comme par exemple "il faut savoir que"). Puisqu'il incombe à l'exposition de mettre en place la vraisemblance, un réajustement du réel intervient, notamment à travers la localisation (métonymique) de l'événement extraordinaire: "le récit, pour être récit d'un extraordinaire, doit marquer d'un nom (ou d'une caractérisation) le lieu qu'il assigne à l'événement". ${ }^{15}$ Mais si la localisation donne lieu à l'événement, elle informe aussi: "asile", désignant un lieu particulièrement dramatisé, accentue la vraisemblance de l'extraordinaire, qui de ce fait renoue avec son ancrage dans le réel.

En invoquant la réception du télégramme, le narrateur ne restaure la vraisemblance qu'au seul plan de l'extraordinaire: en citant le texte du télégramme fictif, le narrateur vraisemblabilise l'hésitation sur la localisation temporelle de la mort, au départ de la confusion possible entre le moment de l'événement et celui de sa communication: "Mère décédée. Enterrement demain. Sentiments distingués".

Peu à peu, le sens s'organise autour du télégramme, et avec lui, l'enchaînement causal qui s'établit rétrospectivement: l'omission de la date du décès (mère décédée...) est au fondement de cet affolement du récit, par où il semble ne pouvoir sortir de sa formulation initiale. L'incessante correction de l'incipit s'avère n'être que la correction d'un texte extérieur à la narration: en effet, l'énoncé premier ne référait pas à l'événement, à l'extraordinaire en tant que tel, mais le visait à travers la médiation de son annonce.

Le récit s'est donc ouvert sur une brève paralipse, dans la mesure où il n'est pas fait mention de cette donnée: omission latérale qui repose sur un blanc, une lacune (future) comblée d'avance par cette imposture qui, s'avisant de son outrance, cherche à se corriger et n'aboutit qu'à faire circuler les marqueurs "paramétriques". Paradoxalement, le texte dégage de son aporie même son effet de réalité: c'est parce qu'en lui subsiste quelque chose d'inachevé qui le fait correspondre au réel - quoi de plus "naturel" que le narrateur hésite sur une information qu'on ne lui donne pas - qu'il donne l'illusion d'être vrai, trouvant dans son inadéquation même le principe de son prolongement.

Il apparaît donc que l'énoncé premier trompe sur sa place, omet son rapport au discours dont il est lecture, s'affiche comme libre émission ponctuelle du narrateur, et ne s'instaure que de cette (dis-) simulation. Le texte ne parle qu'autant que son dire est porté par un discours second, qui chronologiquement le précède. 
En cela, cet incipit ne fonctionne plus sur le mode euphorique de la bonne conscience romanesque: se construisant selon un processus de contestation interne, il dénonce l'illusion référentielle, en l'affichant, en même temps qu'il atteste ne pouvoir s'en passer. Il instaure un début problématique, mais le dote de raisons d'être vraisemblables.

Le texte met en scène son référent, qui n'est à son tour que la mise en scène de l'événement, dont la distribution s'opère à travers un double écran textuel: codification télégraphique et discours subjectif. Il s'agit là moins d'occultation que de transformation: le texte romanesque produit son référent obscur (mère décédée...) afin qu'en dérive l'éclaircissement. Le texte ne s'affiche comme différence et nécessité qu'en feignant d'établir des rapports avec ce qui n'est pas lui. Plus encore, il ne parvient à se dire comme différence qu'en différant, qu'en écartant provisoirement ce dont il est discours. Le texte feint de reproduire le télégramme sur le mode de la citation - il l'atteste sans l'assumer - alors qu'il le produit comme référence fictive dans le seul but d'y reporter la cause de son dysfonctionnement: "la feinte qu'il produit est précisément feinte". ${ }^{16}$ Il la produit littéralement comme pré-texte à son fondement: c'est une feinte fictive qui lui permet de se faire passer pour vrai, dans la mesure où le texte-référent n'a d'autre fonction que de vraisemblabiliser le doute qui régit le texte premier, dans le même temps qu'il authentifie l'extraordinaire. Tout se passe comme si, incapable de produire ses propres conditions d'énonciation, le texte cherchait son prétexte hors de lui-même, trouvant en ce dehors fictif matière à se fonder en nécessité.

Cautionné par un discours lui-même fictif (et qu'en retour il garantit), mais dont l'effet de réel dérive de la mimésis du style télégraphique, le texte y trouve sa motivation, s'y autovérifie. Appuyé par ce discours second, "le texte peut agir sur le plan dérobé qui est le sien". ${ }^{17}$

Le narrateur commente le discours institutionnalisé sur le décès, en le transposant en discours subjectivé, déritualisé, redistribuant et transformant du même coup des unités prélevées sur un texte extérieur/antérieur, qui s'entame de la même façon (mère: maman, décédé: morte).

Le texte, produisant son prétexte en feignant de le reproduire, ne le produit qu'en le transformant, relance l'information initiale, la varie et l'occulte. Mais si l'on ne parvient à savoir ce qui fait mystère, du moins en a-t-on appris la raison. Poursuivant moins une absence qu'il ne la rend plausible, le récit reprend, sans avoir pour fin le dénouement de cette énigme: "Cela ne veut rien dire". L'intervention du narrateur n'est en ce sens motivée que dans la mesure où elle est propre à corriger/annuler une information jugée insuffisante, car si cela ne veut rien dire, cela dit - par extension et de manière d'autant plus 
saillante - la raison pour laquelle il y avait hésitation possible. Par ce retour à l'instance d'énonciation, le narrateur se dessaisit ouvertement de sa responsabilité, dans le même temps où il annule ce qu'il a lui-même invoqué.

Le récit détruit le télégramme, dont le non-sens est son sens à lui, parce que ce n'est que de l'insignifiance de ce qui le cautionne que le récit a pouvoir de mettre en (lieu et) place (de ce discours second). La lecture du télégramme fictif effectuée par le récit ne s'accrédite pas d'une lisibilité accrue, et ne sert qu'à fonder objectivement le tournoiement sur place du texte; une fois garanti par lui, le texte annule son référent, pour renouer avec lui-même: "C'était peut-être hier". En quoi le récit fait retour sur lui-même, revient ouvertement sur ses propres traces, oppose à la citation du référent sa propre citation, à cette différence près qu'il efface l'alternative "ou" pour ne maintenir qu'un doute dès lors légitimé, un flottement orienté. Dénué dès le départ de tout de toute possibilité de dénouement, le texte déroule une ligne qu'il ne peut terminer, se prolonge dans l'ouverture d'une perspective indécidable, qui aussi bien signale sa fermeture, en tant que séquence inaugurale. Dans son effet de redoublement, de recoupement avec la seconde phrase, qu'il répète s'il ne la reproduit pas exactement, cet énoncé referme la boucle ouverte par celle-ci. On y décèle la simulation d'une conclusion: elle porte en elle l'aporie de la démarche, l'impossibilité qu'il y a de finir en elle, ce qui subsiste d'indécidable. Ce n'est que du recouvrement terminal de son origine, que le texte est à même d'induire un effet de cohérence.

De ces retouches incessantes, le texte est fait: l'information initiale est successivement donnée, corrigée, rendue à sa formulation officielle, annulée, puis reprise. Ce processus peu économique n'aboutit finalement pas à l'établissement du dénouement, laisse ouverte l'énigme comme si celle-ci n'était posée que pour relancer le récit sur des voies détournées, désintensifiées de l'extraordinaire. Sans instituer de retour à la vérité, l'incipit s'emploie à se justifier en tant que vraisemblance, selon un processus de reconstruction qui ne mène nulle part, mais s'épuise dans son auto-citation, et s'y clôt. C'est "une vraisemblabilisation qui mime sa production". ${ }^{18}$

Voici donc une fiction qui trébuche sur le premier mot qu'elle produit: ce par quoi elle commence s'avère n'être qu'un lieu vide, qui ne se comble que de l'indécidable, si ce n'est d'arbitraire: lieu vide, emplacement dé-marqué, puisqu'il ne s'établit que métonymiquement, sur la base d'une substitution: "variations sur un incipit" qui fait défaut.

Alors que la logique du récit ouvre sur une infinité de possibles narratifs parmi lesquels il doit s'affirmer, c'est au niveau de l'instance narrative même que se situe la difficulté de commencer: le narrateur diverge sans cesse de son 
propos, ne l'assume pas, s'en défait. Avec l'effondrement de la désignation temporelle sur laquelle s'appuie le narrateur, celui-ci ne peut au mieux que se dessaisir d'un discours qu'il n'est pas en mesure d'assurer.

Finalement, la séquence (voire le parcours) d'initiation ne progresse que vers sa fin qu'en concrétisant sa propre inutilité, puisque le récit se rapporte à un discours dont il ne se sépare que peu, celui du télégramme fictif. S'affiche ainsi une conception de la littérature où le manque, l'incomplétude sont l'indice par lequel le récit signale sa propre déconstruction. L'énoncé premier, dans le même temps où il est formulé, paradigmatise - non moins incongrûment qu'expressément - une reprise contradictoire de lui-même. A la différence du roman euphorique, les contradictions sont ouvertement maintenues, l'énigme n'étant point résolue. Voir la fiction dans son décentrement effectif, en dégageant ce qui en elle est absence déterminée, c'est être amené à la considérer comme fondée sur un manque, un zéro, sans lequel toutefois elle ne serait pas: "le récit n'existe que temporalisé". ${ }^{19}$ En discernant le statut nécessaire du non-dit dans le texte, il apparaît que le récit ne détient la parole que d'un silence, ne s'instaure que de la présence d'un manque, ne parvient à se dire que pour autant qu'un non-dit, et plus encore un rien-à-dire, y surgisse. Pivotant autour de cette lacune qu'il ne peut combler, le récit dès lors la rend visible, détourne ainsi le regard du déjà-dit (le décès): en désignant un leurre, il cache l'extraordinaire.

A peine établi, le récit se met à virer, se détourne de sa programmation initiale: il se met à vivre à rebours de lui-même, la manie interprétative recouvrant l'histoire et la comblant, en ce manque qui ne devient visible que régressivement; il se parcourt à l'envers, en vue d'une issue dérisoire derrière laquelle il n'y a rien, puisqu'il n'avance que pour mieux reculer. De ce déboîtement qui le lie à son double référentiel, le récit finit par s'enrouler sur lui-même, donnant l'illusion de sa cohérence, en instaurant une perspective infinie que figure le maintien du doute.

Incorporant dans son déroulement le modèle auquel il réfère, le récit le corrige, le modifie: il n'avance que pour renouer avec ce qui l'a (ce qu'il a) suscité. Ainsi le narrateur récuse-t-il la responsabilité de prendre sur lui l'incipit dans ce qu'il a de tranchant et de décisif. Il s'introduit subrepticement, se glisse sous le texte, et plutôt que de prendre la parole, il se dessaisit: au moment de parler, qui est le moment d'effacer tout discours du réel pour se resserrer sur un réel du discours, il enchaîne sur un autre discours, fonctionnant littéralement comme "prose du monde venant trouer le texte", ${ }^{20}$ ' y accroche, le poursuit et le complète.

On ne peut, à proprement parler, signaler de commencement; le discours est doublé d'avance. Plutôt parlerons-nous de parole prolongeant un discours 
d'autrui, pour y mettre un sens nouveau. "Celui qui écrit est le même qui lit. Son interlocuteur étant un texte, il n'est lui-même qu'un texte qui se relit en se réécrivant". 21

Le simulacre inaugural du texte porte lui-même sur une feinte plus radicale encore, qui est de manifester sa progressive reconstruction: le texte expose son processus de constitution, exhibe l'enchevêtrement qui le suscite. Simulacre d'autant plus signifiant que cet incessant mouvement de fin et de recommencement prévoit et sous-tend la structure fondamentale qui régira tout le récit romanesque. Or, si les premières lignes renseignent "d'entrée de jeu" le lecteur sur le déclenchement d'un statut problématique de "glissement", de "démenti", et de "flottement", phénomènes provoqués tous, dès l'ouverture, par une espèce de travestissement sitôt déjoué, un tel "tournoiement sur place" éclaire du même coup le processus narratif qui - il faut y succomber sur-déterminera le roman intégral. Car la suite découlera justement du paradigme initial, de ce soi-disant univers fictionnel de l'indécidable produit par le biais de ces "variations sur un incipit". Le texte constitue, en fin de compte, une inépuisable reprise - implicite, ludique, parfois insidieuse - du même et seul commencement. Or, la matrice (dé-) génératrice qui se forme et qui s'élabore dès la phrase d'ouverture présidera, sans répit, jusqu'au dernier mot de l'écrit. C'est un texte destiné à hésiter, à flotter, un texte qui résiste à vouloir dire et dont la résistance ne cesse expressément de résonner. Perfide guet-apens: qu'on ne se laisse pas prendre au piège.

C'est ce qui fait de lui ce texte troué, dé-cipité, si l'on peut dire, revenant inlassablement sur la même chose, feignant de ne rien dire qui ne soit déjà dit, puisque aussi bien il n'est fait que pour se dire soi-même.

Si les présents propos, portés qu'ils sont par l'institution littéraire, entourent cet incipit d'une attention particulière, et lui imposent tout un rituel, le narrateur désire n'avoir pas à commencer, désire s'installer d'emblée dans un déjà-commencé, feint de se reposer sur un discours lui venant de l'extérieur. Mais, comme incongrûment, ce discours qui lui permet de débuter (et ne lui permet que cela), se révèle celui, le même, qui lui interdit de finir. Or l'achèvement de l'incipit assure sa propre pérennité, opère un processus de perpétuel recommencement. ${ }^{22}$ Curieuse dynamique, enfin. 


\section{NOTES}

(1) Nous ne limitons pas l'analyse de l'incipit à la seule première phrase, ainsi que l'envisagent et le circonscrivent Claude Duchet, "Pour une socio-critique ou variations sur un incipit", Littérature 1 (1971); et, quoique dans un cadre plus étendu, moins rétréci, Steven G. Kellman, "Of grand openings and plain: the Poetics of first lines", SubStance (1980). Le découpage est, en fait, fonction d'une unité de récit, dont les critères divergent inéluctablement selon des principes définitionnels. Or dans le cas de L'etranger, nous nous bornons au premier retour du récit sur lui-même, qui manifeste de manière signifiante un effet de division et de clôture.

(2) Charles Grivel, Production de l'intérêt romanesque (La Haye-Paris: Mouton, 1973).

(3) Grivel, p. 93. Dans une autre optique, autre mais bien pertinente, on consultera à profit un certain nombre de travaux consacrés à l'étude du paratexte (multiplement défini et élaboré): Richard-Laurent Barnett, (Ed.), La préface: analyses opératoires. Numéro spécial de L'Esprit Créateur 27 (1987); Gérard Genette, Palimpsestes: La littérature au second degré (Paris: Editions du Seuil, 1982) et Seuils (Paris: Editions du Seuil, 1987); Henri Mitterand, "Le discours préfaciel" dans La lecture socio-critique du texte romanesque. G. Folconer et H. Mitterand. (Ed.) (Toronto: A.M. Hakkert, 1975), p. 3-13; F. Rigolot, (Ed.), Le paratexte. Numéro spécial de SubStance (1987); et Edward Said, Beginnings, intention and method (Baltimore: The Johns Hopkins University Press, 1978). Said propose de reconnaître trois conditions principales à la genèse du récit fictif et voit dans le discours liminaire la compensation d'une "peur du vide" (cf. Walter Benjamin). Le préfacier se sent obligé de fonder son discours sur le vraisemblable plutôt que sur le vrai ( $c f$. Aristote).

(4) Albert Camus, L'etranger (Paris: Gallimard, 1957), p. 7. L'oeuvre camusienne faut-il le souligner - a suscité une véritable pullulation d'analyses dont certaines ne sauraient échapper à l'attention, car elles touchent à nos propos: Jean Gassin, L'univers symbolique d'Albert Camus (Paris: Minard, 1981); Jean Grenier, Albert Camus: soleil et ombre (Paris: Gallimard, 1987); Alain Costes, Albert Camus et la parole manquante (Paris: José Corti, 1973); J. Lévi-Valensi, (Ed.), Albert Camus et les critiques de notre temps (Paris: Garnier, 1970); Raymond Gay-Crosier, (Ed.), Albert Camus (Gainesville: University Press of Florida, 1980); Bettina L. Knapp, (Ed.), Critical essays on Albert Camus (Boston: G.K. Hall, 1988); Laurent Mailhot, Albert Camus ou l'imagination du désert (Montréal: Presses Universitaires de Montréal, 1973). Pour de plus amples renseignements sur l'appareil liminaire comme mécanisme opératoire, voir, dans chacune des sources suivantes, les chapitres consacrés à l'oeuvre de Camus: RichardLaurent Barnett, Inoperative tropes: discourses of auto-subversion (Genève: Droz, à paraître); Herméneutique et indicible: micro-lectures interstitielles (Paris: Seuil, à paraître); et La double ablation (Paris: José Corti, à paraître). Consulter également la récente étude de H.M. Julien, "Parcours de l'initiation, parcours poétique", Romanic Review (1998), p. 411-428. 
(5) Grivel, p. 89.

(6) Grivel, p. 97. Quant à la structure du texte telle qu'elle en sur-détermine le sens, voir Jules Brody, "Camus et la pensée tragique: L'etranger", Saggi e Ricerche di Letteratura Francese (1976), p. 203-241.

(7) Grivel, p. 97. Cette notion est amplement explorée par Michael Riffaterre, Essais de stylistique structurale (Paris: Flammarion, 1970); Semiotics of poetry (Bloomington: Indiana University Press, 1978); Production du texte (Paris: Seuil, 1979).

(8) Selon qu'on lit "est morte" comme état présent (être mort) ou comme action révolue (mourir), l'incipit annonce aussi bien un discours biographique qu'un récit auto-biographique.

(9) Non pas celui qui parle. On le sait, Camus joue sur un autre extraordinaire que le malheur: le fait que la mort n'en soit pas un, en l'occurrence. Voir, à cet égard, Richard-Laurent Barnett, "Sémiotique de l'absence", Travaux de Littérature (1996), pp. 174-189; "Nothing but difference: of poetic rescission in Camus' La peste", Symposium (1987), p. 1-14; "The trope disfigured: effacement and epidemicity in Camus' La peste", Rivista di Letterature Moderne e Comparate (2000), p. 18-37; et "Indices préambulaires", Revue de Litterature Comparée (2000), p. 166-184.

(10) Grivel,p. 155. Consulter, sous ce rapport, Roland Barthes, Le degré zéro del'écriture (Paris: Editions du Seuil, 1953) et Essais critiques (Paris: Editions du Seuil, 1964) ainsi que l'étude de Brian T. Fitch, The narcissistic text: a reading of Camus' fiction (Toronto: University of Toronto Press, 1982), étude qui approfondit de manière perspicace et nuancée les "codes intra-textuels" du roman camusien.

(11) Grivel, p. 157. Quant aux "opérations" de la mimésis, consulter, entre autres, John D. Boyd, The function of mimesis and its decline (New York: Fordham University, 1980); M.H. Abrams, The mirror and the lamp (New York: Oxford, 1953); J.D. Lyons and Stephen J. Nichols, (Eds.) Mimesis: from mirror to method (Hanover: University Press of New England, 1982); Christopher Prendergast, The order of mimesis (Cambridge: Cambridge University Press, 1986); Richard Rorty, Philosophy and the mirror of nature (Princeton: Princeton University Press, 1979); Mihai Sparisou, (Ed.), Mimesis in contemporary theory (Philadelphia: John Benjamins, 1984); Jerry Varsava, Contingent meanings, postmodern fiction, mimesis and the reader (Tallahassee: Florida State University Press, 1990); M.J. Muratore, Mimesis and metatextuality (Genève: Droz, 1994).

(12) Emile Benveniste, Problèmes de linguistique générale, I (Paris: NRF), p. 224.

(13) Duchet, p. 13. Voir aussi Poétique de la prose (Paris: Seuil, 1977); Roland Barthes, Critique et vérité (Paris: Seuil, 1966); Ross Chambers, Story and situation (Minneapolis: University of Minnesota Press, 1984); Gérard Genette, Figures III (Paris: Seuil, 1972); Soren Kierkegaard, Repetition (Princeton: Princeton University Press, 1941); ainsi que l'étude à la fois brève et magistrale de Michael Riffaterre, "Semiotique du récit", Etudes de Lettres 44 (1997), p. 41-68. 
(14) Grivel, p. 91.

(15) Grivel,p. 102. Il faut remarquer ici que le texte bloqué sur le plan temporel repartira sur des coordonnées spatiales: "L'asile de vieillards est à Marengo, à quatre-vingts kilomètres d'Alger".

(16) Grivel, p. 89. Cette notion, diversement entamée depuis les années 60, est utilement reprise et renchérie par Gerald Graff, Literature against itself (Chicago: Chicago University Press, 1979); et - nul besoin de l'affirmer - par Jacques Derrida, La dissémination (Paris: Seuil, 1972) et Positions (Paris: Minuit, 1972).

(17) Grivel, p. 30.

(18) Julia Kristeva, Le texte du roman (La Haye-Paris: Mouton, 1970), p. 77. Cette notion d'auto-annulation, ainsi que nous la propose Kristeva, donne essor à une toute récente étude consacrée au théâtre néo-classique: Richard-Laurent Barnett, "Subversion et défaite textuelles: l'anti-mimétisme cornélien”, Studi di Letteratura Francese (1999), p. 194-228.

(19) Grivel, p. 98. On consultera, toujours à profit, l'ensemble de lectures pénétrantes (et durables) que nous offre Georges Poulet, Etudes sur le temps humain (Paris: Plon, 1950); et, quoique sous une toute autre optique, les observations approfondies promulgées par H. White, Temporality and literature: intersections and departures (Baltimore: The Johns Hopkins University Press, 1988).

(20) Duchet, p. 8.

(21) Kristeva, Le Texte du roman, p. 94. Pour reprendre une autre perspective, citons Aragon: "Pour moi, tout début (les phrases surgies, dictées) d'où je pars vers quelque chose qui sera le roman, au sens illimité du mot, a ce caractère de carrefour, sinon entre le vice et la vertu, du moins entre se taire et dire, entre la vie et la mort, entre la création et la stérilité". Louis Aragon, Je n'ai jamais appris à écrire, ou les incipits (Genève: Skira, 1970). Formule re-citée par Raymond Jean, "Ouvertures, phrases, seuils", Critique 288 (1971), p. 423, et renchérie encore par M. Fumaroli, "Le devant du début", Critique (1996), p. 331-367.

(22) Cet essai de synthèse eût-il été réalisé sans le long entretien méditatif dont Lucien Dällenbach me fit l'amitié en 1999, à Genève? Trop rare privilège. 


\section{RÉSUMÉ}

Le texte de L'étranger constitue, en fin de compte, une inépuisable reprise implicite, ludique, parfois insidieuse - du même et seul commencement. Or, la matrice (dé-) génératrice qui se forme et s'élabore dès la phrase d'ouverture présidera sans répit jusqu'au dernier mot de l'écrit. C'est un texte destiné, par extension, à hésiter, à flotter, un texte qui résiste à vouloir dire - alors qu'il s'arroge le vertige de son inadmissible forclusion - et dont la résistance ne cesse expressément de résonner. Perfide guet-apens: qu' on ne se laisse pas prendre au piège.

Mots-clé: Albert Camus, L'étranger, poétique de la prose, avant-récit, narratologie, sémiotique.

\section{RESUMO}

$O$ texto de $O$ estrangeiro constitui, no fim de contas, uma inesgotável retomada - implícita, lúdica, por vezes insidiosa - de um único e mesmo início, pois a matriz (de-) ge(ne)radora que se forma e se elabora a partir da primeira frase será determinante até a última palavra da narrativa. É um texto destinado, por extensão, a hesitar, a flutuar; um texto que resiste a significar enquanto se permite a vertigem de sua inadmissível exclusão, e cuja resistência não cessa claramente de ressoar. Pérfida emboscada: não nos deixemos cair na armadilha.

Palavras-chave: Albert Camus, $\mathrm{O}$ estrangeiro, poética da prosa, avant-récit, narratologia, semiótica.

\section{OUVRAGES CITÉS}

ABRAMS, M.H. The mirror and the lamp. New York: Oxford University Press, 1953. ARAGON, Louis. Je n'ai jamais appris à écrire, ou les incipits. Genève: Skira, 1970.

BARNETT, Richard-Laurent. Herméneutique et indicible: parcours interstitiels. Paris: Seuil, 2001.

. Imperiled representation: discourses of auto-subversion. New Orleans: University Press of the South, 2001.

. Indices préambulaires. Revue de littérature comparée (2000). . La double ablatione. Paris: José Corti, 2000. 
(1987).

(Éd.). La préface: analyses opératoires. Numéro spécial de L'Esprit créateur $\overrightarrow{(1987)}$.

Nothing but difference: of poetic rescission in Camus' La peste. Symposium Poétique des marges: lectures d'excentration. Paris et New Orleans: Presses Universitaires du Nouveau Monde, 2001.

. Sémiotique de l'absence. Travaux de littérature (1996).

. Subversion et défait textuelles: l'anti-mimétisme cornélien. Studi di letteratura francese (1999).

. The trope disfigured: effacement and epidemicity in Camus' La peste. Rivista di letterature moderne e comparate (2000).

BARTHES, Roland. Critique et vérité. Paris: Seuil, 1966.

. Essais critiques. Paris: Seuil, 1964.

Le degré zéro de l'écriture. Paris: Seuil, 1953.

BENVENISTE, Emile. Problèmes de linguistique générale. Paris: NRF, 1971.

BOYD, John D. The function of mimesis and its decline. New York: Fordham University Press, 1980.

BRODY, Jules. Camus et la pensée tragique: L'Étranger. Saggi e ricerche di letteratura francese (1976).

CAMUS, Albert. L'Étranger. Paris: Gallimard, 1957.

CHAMBERS, Ross. Story and situation. Minneapolis: University of Minnesota Press, 1984.

COSTES, Alain. Albert Camus et la parole manquante. Paris: José Corti, 1973.

DERRIDA, Jacques. La dissémination. Paris: Seuil, 1972.

. Positions. Paris: Minuit, 1972.

DUCHET, Claude. Pour une socio-critique ou variations sur un incipit. Littérature (1971).

FITCH, Brian T. The narcissistic text: a reading of Camus' fiction. Toronto: University of Toronto Press, 1982.

FOLCONER, G. (Éd.). La lecture socio-critique du texte romanesque. Toronto: A. M. Hakkert, Ltd., 1973.

FUMAROLI, MARC. Le devant du début. Critique (1996).

GASSIN, Jean. L'univers symbolique d'Albert Camus. Paris: Minard, 1981.

GAY-CROSIER, Raymond (Éd.). Albert Camus. Gainesville: University Press of Florida, 1980. 
GENETTE, Gérard. Figures III. Paris: Seuil, 1972.

Palimpsestes: la littérature au second degré. Paris: Seuil, 1982. Seuils. Paris: Seuil, 1987.

GRAFF, Gerald. Literature against itself. Chicago: University of Chicago Press, 1979. GRENIER, Jean. Albert Camus: soleil et ombre - Paris: Gallimard, 1987.

GRIVEL, Charles. Production de l'intérêt romanesque. La Haye - Paris: Mouton, 1973. JEAN, Raymond. Ouvertures, phrases, seuils. Critique (1996).

JULIEN, H.M. Parcours de l'initiation, parcours poétique. Romanic Review (1998).

KELLMAN, Steven G. Of grand openings and plain: the poetics of first lines. SubStance (1980).

KIERKEGAARD, Soren. Repetition. Princeton: Princeton University Press, 1941.

KNAPP, Bettina L. (Éd.). Critical essays on Albert Camus. Boston: G.K. Hall, 1988.

KRISTEVA, Julia. Le texte du roman. La Haye-Paris: Mouton, 1970.

LÉVI-VALENSI, J. (Éd.). Albert Camus et les critiques de notre temps. Paris: Garnier, 1970.

LYONS, J.D.; S.J. Nichols. (Éds.). Mimesis from mirror to method. Hanover: University Press of New England, 1982.

MAILHOT, Laurent. Albert Camus ou l'imagination du désert. Montréal: Presses de l'Université de Montréal, 1973.

MITTERAND, Henri. Le discours préfaciel dans G. Folconer, (Éd.), La lecture sociocritique du texte romanesque. Toronto: A.M. Hakkert, Lid., 1975.

MURATORE, M.J. Mimesis and metatextuality. Genève: Droz, 1994.

POULET, Georges. Etudes sur le temps humain. Paris: Plon, 1950.

PRENDERGAST, Christopher. The order of mimesis. Cambridge: Cambridge University Press, 1986.

RIFFATERRE, Michael. Essais de stylistique structurale. Paris: Flammarion, 1970. . Production dlutexte. Paris: Seuil, 1979. . Semiotics of poetry. Bloomington: Indiana University Press, 1978. . "Sémiotique du récit". Etudes de lettres (1997).

RIGOLOT, F., (Éd.). Le paratexte. Numéro spécial de SubStance (1987).

RORTY, Richard. Philosophy and the mirror of nature. Princeton: Princeton University Press, 1979.

SAID, Edward. Beginnings, intention and method. Baltimore: The Johns Hopkins University Press, 1978. 
SPARISOU, Mihai, (Éd.). Mimesis in contemporary theory. Philadelphia: John Benjamins, 1984.

TODOROV, T. Poétique de la prose. Paris: Seuil, 1977.

VARSAVA, J. Contingent meanings, postmodern fiction, mimesis and the reader. Tallahassee: Florida State University Press, 1990.

WHITE, H. Temporality and literature: intersections and departures. Baltimore: The Johns Hopkins University Press, 1988. 